\title{
Electrical Contacts and Gas Sensing Analysis of Individual Metal Oxide Nanowires and 3-D Nanocrystal Networks
}

\author{
Francisco Hernández-Ramírez Non-member \\ Albert Tarancón \\ Non-member \\ Albert Romano-Rodríguez \\ Non-member \\ J. R. Morante \\ Non-member
}

\begin{abstract}
A nanolithography method based on the use of a Dual Beam focused-ion-beam (FIB) equipment to perform electrical contacts on either individual metal oxide nanowires or three-dimensional (3-D) nanocrystal networks is reported. Both advantages and disadvantages of using this nanolithography process compared with other more conventional techniques are discussed. The possibility of using these FIB bottom-up devices in gas sensing application is also presented showing the performances of the gas sensors based on single nanowires and 3D nanocrystal networks of metal oxides. For it, two- and four-probe electrical measurements have been used determining the features associated to the contact resistances. To eliminate any influence of the contact values, AC Impedance Spectroscopy techniques have been adapted on individual nanowires facilitating the analysis of the gas sensing mechanisms in single metal oxide nanocrystal.
\end{abstract}

Keywords : Metal-oxide nanowire, 3D-nanocrystal network, Focused ion beam FIB, Gas sensor, AC impedance spectroscopy

\section{Introduction}

Nanomaterials represent the natural bridge between single molecules and macroscopic bulk materials. Their finite size and limited number of electronic states are arisen a lot of boundaries for the transport mechanisms, specially, for the charge conduction as the surface state density is comparable with that of the bulk. So, their electrical behavior is expected to present novel electrical and optical properties which can be used to fabricate nanodevices with improved capabilities ${ }^{(1)}$.

Among all nanomaterials, metal oxides nanowires and three-dimensional (3D) nanocrystal networks are one of the most promising ones ${ }^{(2)}$. Unlike to the small quasi spherical nanoparticles with not faceted surfaces, these nanosystems are presenting well defined crystallographic directions as crystal surfaces as well as they look like a perfect monocrystal. No grain boundaries are present. Therefore, the electrical transduction effects induced by the adsorbed gas molecules onto the surface of these nanosystems can straightforward be revealed by the electrical performances of these single nanostructures. Moreover, as the gas molecules adsorption takes place at the surface, one of the most important issues for tailoring the sensor material response is the control of its active surface area. Many authors ${ }^{(3)}$ have shown that there is a direct relationship between the active surface areas presented by the sensing material and the response given by the macroscopic sensor fabricated with these nanomaterials. According to these results, an increase of the surface area should involve an increase of the sensor response. This is the case of nanowires and 3D nanocrystal networks, where the area-volume ratio is easily maximized as the diameter size is decreased.

EME/CeRMAE/IN ${ }^{2} U B$, Departament of Electronics, Physics Faculty, University of Barcelona. C/ Martí I Franquès 1. Barcelona 08028. Spain
In these framework, the majority of the experimental works show gas sensor based on multiples nanostructure. A large number of these nanostructures are contacted and their electrical parameters estimated. However, only some equivalent mean values of these parameters are determined due to the dispersion existing among the contacted nanostructures and the grain boundaries among them. In this scenario, it is not straightforward to study the sensing mechanisms onto a single metal oxide nanocrystal.

To study gas sensing mechanism onto one individual nanocrystal is still a challenge. It is required for reaching a better knowledge of the electrical transport mechanisms which take place in these nanostructures. One of the reason to explain this lack of experimental studies is that there are many difficulties in performing reliable electrical contacts on one individual nanostructure in a controllable fabrication process at the nanoscale level.

The most common techniques are optical- or electron-beam lithographies. Nevertheless, both techniques are multi-step and time consuming processes ${ }^{(4)(5)}$ and they are difficult to tailor for each specific sample. For all these reasons, an alternative lithography process based on FIB technique is presented here, as one option which could, experimentally, help to solve problems related to conventional lithography processes.

Focused Ion Beam (FIB) is a powerful technique developed during the late 1970 s and the early 1980s for the patterning and, later, for the deposition of materials, with resolution in the tens of $\mathrm{nm}$ range, and commonly used in circuit edit, mask repair, microsystem technology processes and material characterisation (6)-(11). The basic principle of this technique is a focused ion beam of highly energetic particles that scans the sample's surface and sputters the material of the exposed area. The scanning can be performed, similarly to a SEM, using electrostatic lenses and, thus, the milling occurs without the need of masks. At the present time, 
gallium $\left(\mathrm{Ga}^{+}\right)$ions are the most used particles in FIB technique, due to the fact that gallium is a metallic element with a low melting temperature which allows the fabrication of long life-time, high brightness and reliable liquid metal ion source (LMIS) required in FIB technique. Moreover the element gallium is positioned in the centre of the periodic table (element number 31), so its momentum transfer capability is optimal for a wide variety of materials. On the contrary, lighter elements would be less efficient in milling heavier elements ${ }^{(12)}$.

On the other hand, if a metalorganic compound is introduced in the beam path with the help of a so-called gas delivery system by using a microneedle, decomposition occurs due to interaction of the compound with both secondary electrons and ions originated during the $\mathrm{Ga}^{+}$ion bombardment. Part of the compound can be deposited on the sample's surface (ion-assisted deposition) or can reactively assist the milling process (gas assisted etching), while the rest is removed by the vacuum system ${ }^{(5)}$. In this way, conductive and isolating materials can easily be deposited with FIB with nanometer precision ${ }^{(6)}$. Although the purity of the deposition is generally lower as compared to conventional deposition techniques like CVD or PVD, owing to contamination originated during the metalorganic decomposition, the main advantage of this technique is its flexibility due to its direct writing capabilities and because masks are not required ${ }^{(12)-(14)}$.

In the past, other groups have attempted to use FIB lithography to contact nanometre-sized materials. However, damage introduced in these materials by ion bombardment $\mathrm{CGa}^{+}$ions accelerated to $30 \mathrm{kV}$, in our case) during fabrication of contacts has limited the use of this technique ${ }^{(15)-(20)}$. Alternatively, other laboratories have tried to fabricate electrical contacts by using low ion currents in the proximity of the nanomaterials in order to reduce the damage. However, ion exposure is not thoroughly eliminated and contacted nanomaterials are still modified ${ }^{(19)(20)}$.

At the present, the appearance of the so-called Dual Beam systems, a traditional FIB which incorporates a Scanning Electron Microscope (SEM), has facilitated the use of FIB thanks to the possibility of acquiring electron images in-situ, avoiding the damage caused by ions when imaging ${ }^{(21)}$. Moreover, metalorganic compounds can be also dissociated with the help of secondary electrons (SE), giving rise to electron-beam induced deposition $(\text { EBID })^{(22)}$. Nevertheless, the number of SE produced by the primary electrons is smaller than produced by $\mathrm{Ga}^{+}$ions, so the deposition rate is much lower in an electron-beam induced deposition $^{(12)}$.

Due to the fact that interaction between electrons and the sample is less destructive, performing an electron-assisted deposition on the nanostructure to be contacted and finishing the rest of the contact with the help of ions can avoid undesired surface damage and structure modification of the nanostructure. According to this procedure based on the combination of both electron- and ion-assisted deposition, metal oxide nanowires (NWs) and 3D nanocrystal networks can be contacted either in 2or 4-probe configuration. The attempts of contacting nanomaterials using this method are still scarce and further research must be done in order to have a complete knowledge of the overall characteristics and performances of the electrical contacts $^{(23)-(26)}$.

A better understanding of these so-called Dual Beam FIB-nanocontacts is required to avoid false electrical interpretations of the NWs-based on experiments. It is introduced, mainly, by contact resistance underestimations. Moreover, knowing if ohmic contacts can be fabricated is really important, since in case that obtaining such contacts were not feasible, the design of field-effect transistors (FETs) based on a single nanowire would not be possible with this nanolithography technique. On the contrary, the existence of rectifying contacts would allow the measurement of Schottky-barrier transistors based in one single nanomaterial.

In this paper, the possibility of contacting nanomaterials with Dual Beam FIB lithography is presented, and both the quality of the deposited platinum and contacts are evaluated as a function of their dimensions, chemical composition, current density through them and time. The electrical response of some of the contacted NWs and 3D nanocrystal networks is also presented, demonstrating the possibility of extracting their electrical parameters using this nanofabrication technique.

Finally, the use of Dual Beam FIB lithography to fabricate nanodevices, following a bottom-up process, is demonstrated by contacting metal-oxide nanowires and 3D nanocrystal networks as gas sensor devices.

\section{Experimental}

A FEI Strata 235 Dual Beam machine, which works with $\mathrm{Ga}^{+}$ ions accelerated to an energy of $30 \mathrm{keV}$, electrons accelerated to $5 \mathrm{keV}$, and methyl cyclo pentadienyl $\mathrm{Pt}(\mathrm{IV})\left(\mathrm{PtC}_{6} \mathrm{H}_{16}\right)$ try methyl as gas precursor. has been used to perform both ion- and electron-assisted deposition over the surface of $\mathrm{SiO}_{2} / \mathrm{Si}$ substrates with photo lithographically pre-patterned $\mathrm{Ti} / \mathrm{Ni} / \mathrm{Au}$ microelectrodes. Ion beam currents between 10pA and 100pA have been selected in all experiments. Electrical measurements have been performed with help of both microprobes and microbonding using a Keithley Source Measure Unit (SMU) 2400.

Resistances of both ion- and electron-assisted platinum have been evaluated depositing stripes, whose dimensions have been ranged between (height $\times$ width $\times$ length) $0.13 \times 0.17 \times 10 \mu \mathrm{m}$ and $5 \times 5 \times 15 \mu \mathrm{m}$, among 4 gold microelectrodes. Chemical characterization of the deposited platinum has been obtained from Auger Electron Spectrometry (AES) using a PHI 670 equipment.

Different metal oxide materials have been prepared as nanowires for many different laboratories using several technological approaches mainly based on chemical vapour growth routes $^{(27)}$.These nanowires were dispersed over the surface of substrates with pre-patterned microelectrodes before to carried out the nanomanipulation and nanolithography methods. In this paper we will be focused in the application of $\mathrm{SnO}_{2} \mathrm{NWs}$ which is one of the gas sensing material that is extensively considered as a reference. Electron microscopy image and high resolution transmission picture of one of these $\mathrm{SnO}_{2}$ nanowires is shown in the figure $1 \mathrm{a}$ and $1 \mathrm{~b}$. This synthesis procedure allows us to have nanowires with different diameter from $50 \mathrm{~nm}$ to $300 \mathrm{~nm}$ with length higher than $4 \mu \mathrm{m}$.

In parallel, two different $3 \mathrm{D} \mathrm{WO}_{3}$ nanocrystal networks that were obtained as negative replica of two different mesoporous materials used as hard nanotemplate structures have also been dispersed over similar microelectrodes. The two employed silica mesoporous nano templates are: i) the three-dimensional cubic (space group 1a-3d) named KIT-6, which is probably the most complex one presenting a double gyroidal mesostructure with channels running along [100] and [111] directions, defining a three dimensions pore network ${ }^{(28)}$, figure $1 \mathrm{c}$ and $1 \mathrm{~d}$; ii) the two 
dimensional hexagonal structure (space group p6mm) named SBA-15, which shows a simpler and much better known structure, figure 1e.

In order to measure the electrical response of nanowires and 3D nanocrystal networks, the fabricated bottom up gas sensors based on single nanostructures were tested under different combustion gases such as, $\mathrm{O}_{2}, \mathrm{CO}$ and $\mathrm{NO}_{2}$. For it, a Linkam THMS600 chamber that incorporates a heater and a thermocouple was used. The small volume of the chamber ensures that gas is replaced promptly and the dynamic behavior of the gas can be recorded. The gas flow used was $100 \mathrm{ml} / \mathrm{min}$. Gas mixture is obtained by means of mass flow controllers.

\section{Results and Discussion}

3.1 Pt stripes In the present configuration of our FEI Strata 235 Dual Beam machine and using the 10pA ion aperture, platinum stripes with length of several microns and about $100 \mathrm{~nm}$ wide have been fabricated. This value agrees with the data obtained from the literature ${ }^{(7)}$, and demonstrates the possibility of using FIB lithography in the nanometer range. Actually, the

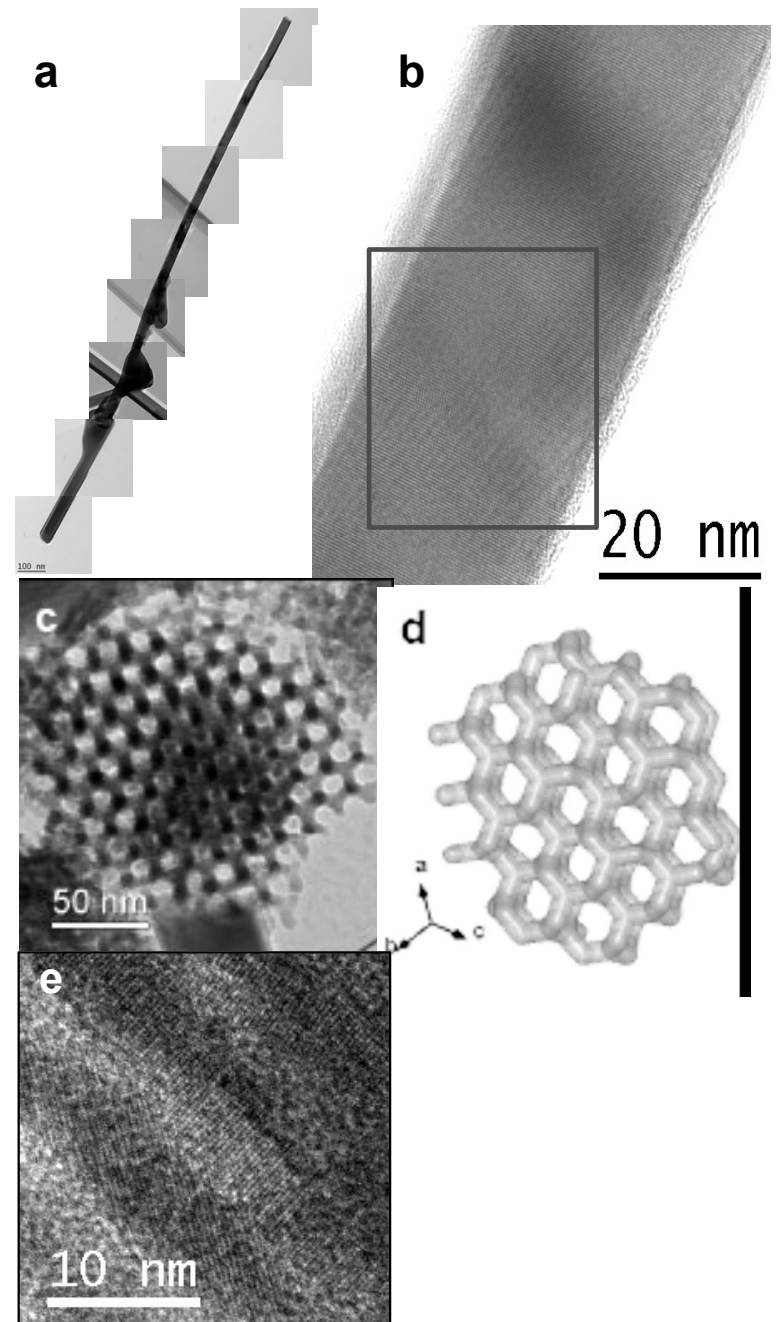

Fig. 1. (a) Electron microscopy image of a $\mathrm{SnO}_{2}$ nanowire. (b) HRTEM picture of a $\mathrm{SnO}_{2}$ nanowire. (c) $\mathrm{WO}_{3}$ nanostructure of a KIT-6 replica. (d) Model of this nanocrystal network. (e) $\mathrm{WO}_{3}$ nanostructure of a SBA-15 replica obtained wide value points out the minimum lateral dimensions of the contact that is possible to fabricate.

Electrical measurements of ion-assisted deposited platinum stripes placed among previously fabricated microelectrodes (Fig.2) have revealed a perfect ohmic behavior. Resistivity values larger than bulk platinum $\left(\rho=1.06 \cdot 10^{-2} \mathrm{~m} \Omega \cdot \mathrm{cm}\right)$, ranging from $\rho=1.5 \mathrm{~m} \Omega \cdot \mathrm{cm}$ for those stripes with cross sections of $5 \times 5 \mu \mathrm{m}^{2}$ to $\rho=5.25 \mathrm{~m} \Omega \cdot \mathrm{cm}$ for those with cross sections of $0.13 \times 0.17 \mu \mathrm{m}^{2}$ (Fig.3) have been found, it means more than 100 times larger than pure platinum.

These resistivity values agree with results previously presented by other groups for platinum ion-induced stripes of different cross-sections $^{(19)(20)}$ and they can be improved applying thermal treatments. In Fig. 3 it can also be observed how the resistivity becomes lower than $\rho=2 \mathrm{~m} \Omega \cdot \mathrm{cm}$ for stripes with cross-sections larger than $1 \mu \mathrm{m}^{2}$. These results are in agreement with the expected FIB characteristics. In the case of cross sections below $1 \mu \mathrm{m}^{2}$, the observed increase of the resistivity could be produced by the decrease of paths for electron conduction and the increase of surface scattering contribution.

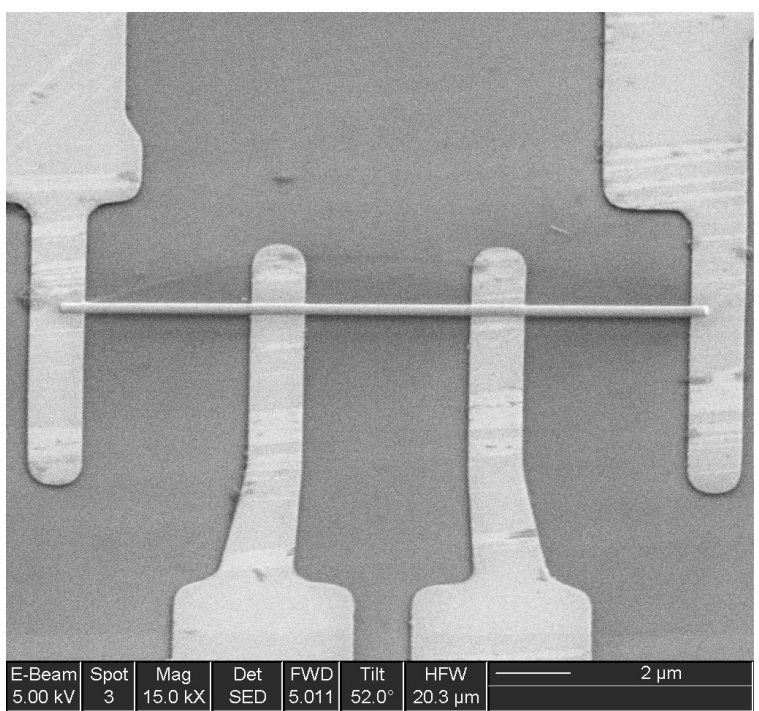

Fig. 2. Platinum ion-beam induced deposition stripe placed among four gold-microelectrodes

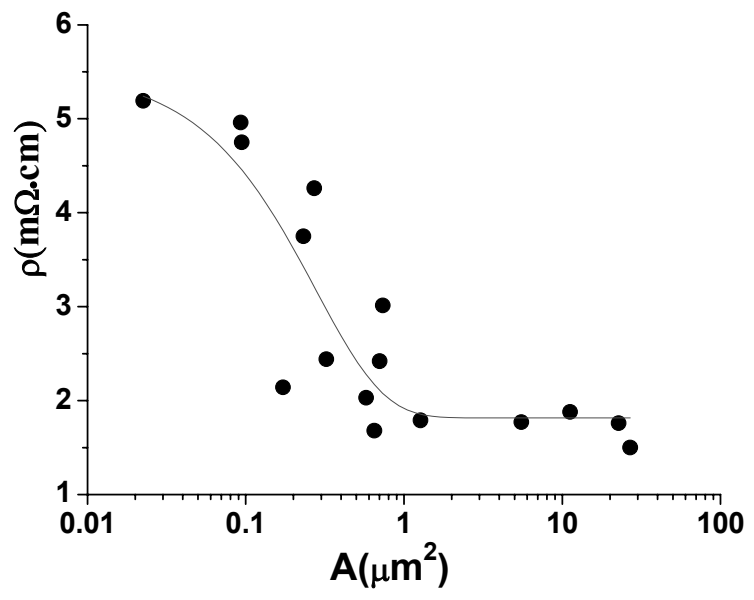

Fig. 3. Resistivity values of ion-assisted deposited platinum stripes in function of their cross-section area 
Auger electron spectrometry (AES) of some of the analysed platinum ion-assisted deposition has revealed a high concentration of $\mathrm{C}$ and some $\mathrm{Ga}$ too $(65 \% \mathrm{C}, 27 \% \mathrm{Pt}, 8 \% \mathrm{Ga})$ originated during the decomposition of the metalorganic compound and by the ion beam effect. It is believed that this high carbon contamination is the responsible of the high resistivity measured before. So, according to these results, it should be more accurate to say that depositions performed with FIB are rather a carbon matrix doped with platinum and gallium.

Contact electrical resistance between platinum ion-assisted deposited stripes and pre-patterned gold microelectrodes has also been evaluated. The measured contact electrical resistance increases with decreasing contact area according to the equation 1.

$$
R_{c}=\rho_{c} / A
$$

Where $R_{c}$ is the contact electrical resistance, $\rho_{c}$ is known as the electrical contact resistivity and A the contact area.

Our data have been fitted to equation 1 , figure 4 , obtaining a value for $\rho_{\mathrm{c}}$ of $210 \pm 20 \Omega \cdot \mu \mathrm{m}$.

Resistance versus current (R(I)) plots of the platinum ion-assisted deposited stripes present a parabolic behavior with decreasing resistance value for increasing intensities. This response is typical of materials with negative temperature coefficient (NTC) when heated. This effect demonstrates that carbon contamination dominates the thermal response of the stripe.

The parabolic behavior described before is reproducible and the resistance variation is instantaneous, demonstrating the rapid variation of the temperature of ion-assisted depositions, due to their small dimensions. No degradation of the uniform and homogenous Pt stripes has been observed with current densities up to $10^{10} \mathrm{~A} / \mathrm{m}^{2}$, equivalent to several tens of microampers. When such value is applied, melting of the stripe begins, finishing with its destruction.

Electrical response of platinum stripes has been evaluated as a function of time and no changes in their resistivity values has been observed even two months after fabrication of the stripes, demonstrating their stability.

In a similar way, the quality of platinum electron-beam induced deposition stripes has also been studied. Ohmic behaviour has

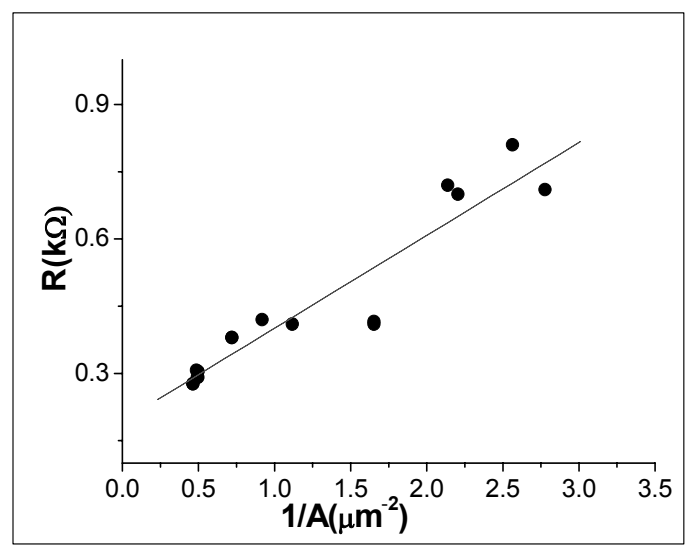

Fig. 4. Contact resistance between ion-assisted deposited platinum stripes and gold microelectrodes as function of the inverse of contact area between them always been found, and a deposition rate much lower than ion-induced deposition observed (between 10 and 100 times). The measured resistivity $(\rho=5.9 \pm 4.0 \Omega \cdot \mathrm{cm})$ is even larger than for ion-assisted deposition, between 100 and 1000 times, and the contact resistance is also much larger, never below $100 \mathrm{k} \Omega$. It is possible that $\mathrm{Ga}$ contamination, which is introduced in ion-assisted deposited platinum by the ion beam, could help to reduce resistivity, working as a doping element ${ }^{(22)}$. R(I) plots of electron-assisted stripes present the same parabolic behavior, demonstrating that its thermal response is also controlled by carbon contamination.

Other electrical parameters like critical current density or stability as function of time are similar for both ion- and electron-beam induced depositions.

3.2 Electrical Nano Contacts on Nanowires and 3D Nanocrystal Networks Considering the above properties, the ion and electron deposited Pt stripes by using the focused ion beam machine can be used to contact single nanostructures previously deposited and nano/micro manipulated onto a silicon wafer already processed to have an adequate distribution of microelectrodes. The manipulation defines a better placement of the nanostructure in front of the microelectrodes before to proceed with the Pt deposition. Then, four or two electron deposited Pt contacts are performed, two or one, respectively, in each extreme. In this way, damage is strongly diminished and $\mathrm{Ga}^{+}$ions effects on the nanostructure are avoided. In fact, a couple of contacts are fabricated near each extreme of the nanostruture requiring much less than a half micron, remaining a significant length of the nanostructure, more than several couples of microns, released of any feature relative to the contact fabrication such as it is shown in the figure 5. Once, the influence area due to the deposition is far away of the nanostructure, ion deposition methodology is used to extend the stripes to the pre-patterned contacts, figure5, because the deposition time is much shorter than using the electron one. The electron- and the $\mathrm{Ga}^{+}$ion beams are accelerated to 5 and 30 $\mathrm{kV}$, respectively.

Using this procedure, the previously presented nanostructures have been contacted in 2- and 4-probe configuration. Both, the electron and the ion beam must be well-aligned in order to prevent the need to take an ion image of the nanowires, which could irreversibly modify them. Monte Carlo's simulations have been performed and indicate that $\mathrm{Ga}^{+}$ions accelerated at energy of 30 $\mathrm{keV}$ have a projected range of $27 \mathrm{~nm}$ in a layer of amorphous silicon, producing damage and sputtering ${ }^{(29)}$. If such distance is assumed to be equal in our nanomaterials, the external layers of these structures would be affected and, hence, it must be avoided in our experiments.

This contact fabrication process for each nanometer-sized material takes more or less two hours, limiting the application of this lithography technique in large scale processes. On the other hand, its flexibility guarantee that it can be used for rapid prototyping, helping to solve some of the multi-step related problems of other nanolithography techniques.

Some times, SEM images of final devices reveal the existence of a bright halo around the contacted NW area. According to previous works ${ }^{(24)}$, this halo is believed to be composed by rests of the metal organic precursor $(\mathrm{C} \& \mathrm{Pt})$ used during the fabrication process. Due to its high expected resistance, the halo effect is not believed to disturb our electrical measurements, since it can be considered as a parallel resistance with an experimental value, 

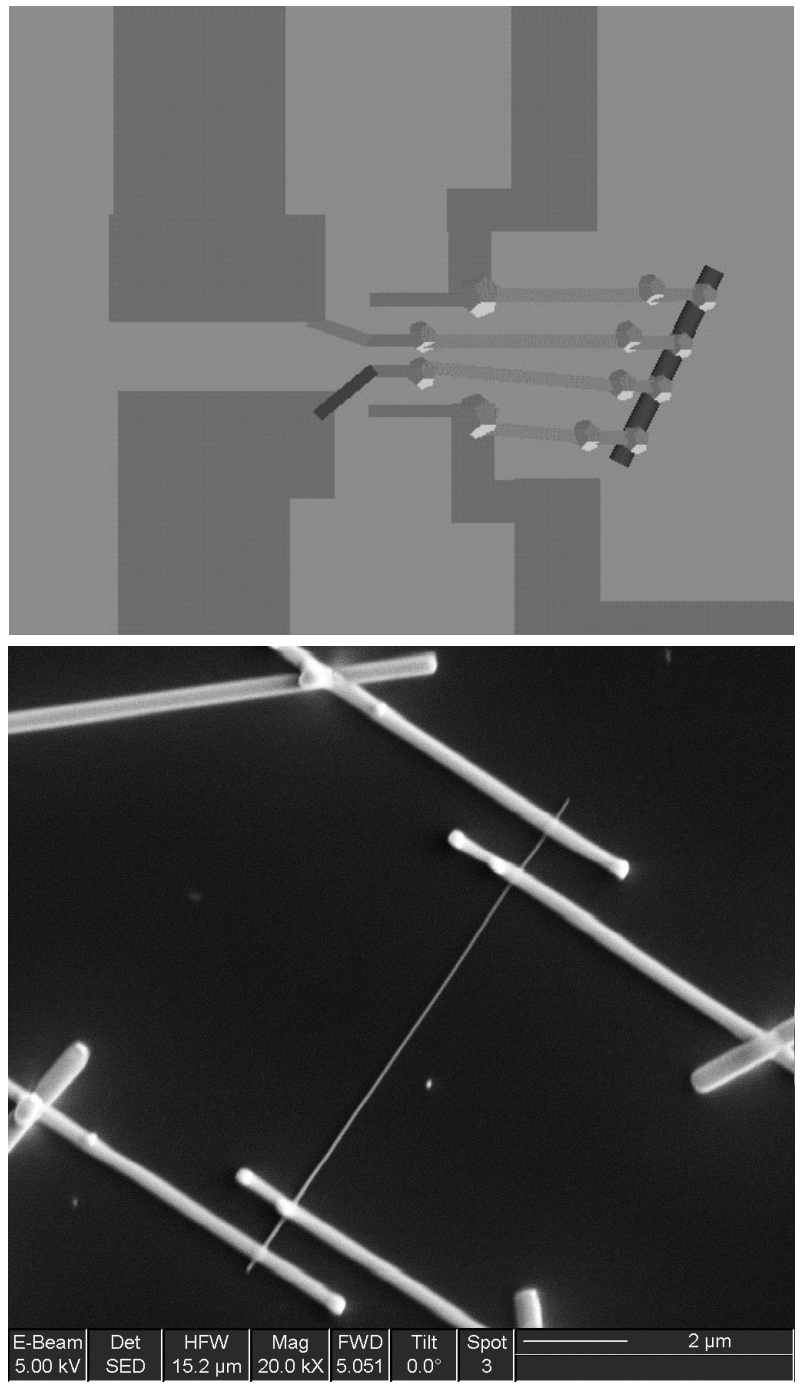

Fig. 5. $\mathrm{SnO}_{2}$ nanowire (length $\mathrm{L}=11 \mu \mathrm{m}$, diameter $\mathrm{D}=55 \mathrm{~nm})$ electrically contacted using Dual Beam FIB lithography technique

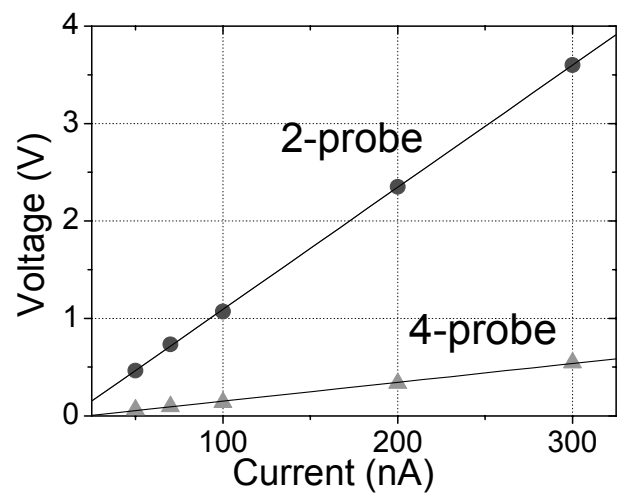

Fig. 6. Two- and four-probe V-I measurements of one $\mathrm{SnO}_{2}$ NW contacted with FIB nanolithography. A resistance of $\mathrm{R}=12,53 \mathrm{M} \Omega$ has been estimated using 2-probe configuration while a resistance of $\mathrm{R}=1,93 \mathrm{M} \Omega$ is found with 4-probe measurements measured in our samples as leakage current, higher than several hundred of gigaohms.

Two- and four- probes V-I electrical measurements have been performed, using a Keithley Source Measure Unit (SMU) 2400. These measurements at room temperature reveal that contact resistance contribution in 2-probe measurements is much more important than the nanowire resistance, figure 6 . In some case, such contribution is even higher than $90 \%$ of total measured resistance, which range between some hundreds of kiloohms and some tens of megaohms. This huge difference between 2- and 4-probe measurements can not be only explained by the sum of FIB-fabricated platinum stripes resistance and the contact resistance between them and pre patterned gold microelectrodes, since according to FIB platinum resistivity, lower resistance values would be expected (a few tens of kiloohms). So, the main part of the measured contact resistance is believed to be originated in the Pt-SnO${ }_{2} \mathrm{NW}$ junction.

No degradation in this kind of contacts has been noticed after long time of their fabrication, more than 4 weeks, and neither after applying constant current for several minutes. For example, good stability of the contacts performed on a nanowire has been observed after 1 month and no degradation after a current of 700nA has been injected during 20 minutes.

All measured nanowires are destroyed at current densities bellow $10^{10} \mathrm{~A} / \mathrm{m}^{2}$, which is the critical current density value obtained for both ion- and electron-assisted deposition.

As it has been said previously, due to the influence of the contact resistance to the final result, 4-probes electrical measurements are required if electrical parameters of nanostructure must be extracted. In order to determine the importance of this contact resistance contribution and as one example, the $\mathrm{SnO}_{2}$ nanowire shown in the figure 5 has been measured in both 2- and 4-probe electrical measurements. A resistance of $\mathrm{R}=12.53 \mathrm{M} \Omega$ has been obtained under the first setup, while a resistance of $\mathrm{R}=1.93 \mathrm{M} \Omega$, see figure 6 , has been obtained in the second case. These results point out clearly how the contribution of the contact impedance distort the measurement of the nanostructure and even, sometimes, can be higher than the resistance of the nanostructure itself.

The extraction of the electrical parameters of the contacted nanowires is performed applying a simple model to estimate the material resistivity. Considering that these nanowires have a cylindrical cross-section, the resistivity can be calculated with the help of equation 2 .

$$
\mathrm{R}=\rho \mathrm{L} / \mathrm{A}=\rho \mathrm{L} / \pi \mathrm{r}^{2}
$$

Where $\mathrm{R}$ is the measured resistance, $\rho$ is the unknown resistivity, $\mathrm{L}$ is the length of the nanowire between the fabricated contacts and A, its cross-section, which can be determined if the radius, $r$, of the nanowire is measured.

Once a nanowire has been contacted, the dimensions $\mathrm{L}$ and $\mathrm{r}$ are measured in the SEM using XP-Annotate $C$ software, which is available in the FIB workstation. This software compensates for the error introduced in the measurements when the sample is tilted.

In order to test the accuracy of this software, morphological characterization has been performed with an AFM working in tapping mode. It has been checked how platinum stripes have a roughness between 2 and $5 \mathrm{~nm}$ and their dimensions differ less than $10 \%$ of those obtained with XP-Annotate $\mathrm{C}$. So, the use of 
this software has been considered a reliable and fast method to determine dimensions ( $\mathrm{L}$ and $\mathrm{R}$ ) of the nanowires.

Once the dimensions and the resistance of the nanowires are known, the resistivity can be easily determined, applying equation 2. In the case of the $\mathrm{SnO}_{2}$ nanowire of figure 5, a resistivity of $\rho=$ $50 \mathrm{~m} \Omega \cdot \mathrm{cm}$ has been found. The found value agrees with the literature related to single $\mathrm{SnO}_{2}$ crystals NWs studies ${ }^{(30)(31)}$.

As a consequence of these characteristics, one can propose this procedure of nano contact fabrication as a reliable method to have electrical access to study the charge transport properties of the nanostructures. Moreover, these contacts show excellent electrical stability as a function of time and applied current density. Therefore, they fulfill the requirements to fabricate nanodevices able to work for a long time. However, there is still an important feature to be discussed. It is the linearity of the electrical contact. Although the data contained in figure 6 correspond to a linear behavior, in many cases the electrical contact with the metal oxide material does not show linear characteristics. These ones are symmetric but reveal a non-ohmic behavior such as it shown in the figure 7.This rectifying behaviour is originated at the $\mathrm{Pt}-\mathrm{SnO}_{2}$ NW junctions, which form a Schottky barrier of height $\Phi_{\mathrm{B}}=0.75 \pm$ $0.10 \mathrm{eV}$ in the absence of interface states, owing to the differences between the work function of $\mathrm{Pt}\left(\Phi_{\mathrm{Pt}}=5.65 \mathrm{eV}\right)$ and the electron affinity of $\mathrm{SnO}_{2}\left(\chi_{\mathrm{SnO} 2}=4.9 \pm 0.1 \mathrm{eV}\right)$. This configuration can be described as a back-to-back Schottky-like circuit.

According to this assumption, the applied bias V must distribute as

$$
V=V_{a}+V_{d}+V_{N W}+V_{i}
$$

where $\mathrm{V}_{\mathrm{a}}$ is the voltage drop in the cables, gold microelectrodes and FIB-fabricated platinum stripes, $\mathrm{V}_{\mathrm{d}}$ is the voltage drop in the direct biased $\mathrm{Pt}-\mathrm{SnO}_{2}$ junction, $\mathrm{V}_{\mathrm{NW}}$ is the voltage drop along the $\mathrm{SnO}_{2} \mathrm{NW}$ and $\mathrm{V}_{\mathrm{i}}$ is the voltage drop in the reverse biased junction. In this back-to-back Schottky-like configuration, the total current is limited by this reverse biased junction, insert in figure 7 . The contact barrier height is reduced and the current increases with increasing bias according to

$$
I=A A^{* *} T^{2} \exp \left(\frac{-q \phi_{B E}}{k_{B} T}\right)
$$

where

$$
\phi_{B E}=\phi_{B 0}-\sqrt{\frac{q E}{4 \pi \varepsilon_{s}}}-\frac{q}{\varepsilon_{S}} \sqrt{\frac{N_{S} \cdot d}{4 \pi}}
$$

and

$$
E=\sqrt{\frac{2 q N_{D}}{\varepsilon_{s}}\left(V+\phi_{b i}-\frac{k_{B} T}{q}\right)}
$$

where $\mathrm{A}$ is the contact area, $\mathrm{A}^{* *}$ is the effective Richardson constant, $\Phi_{\mathrm{BE}}$ is the effective barrier height, $\Phi_{\mathrm{Bo}}$ is the ideal barrier height in the absence of image force, $\mathrm{E}$ is the maximum electric field at the junction, $\varepsilon_{\mathrm{s}}$ and $\mathrm{N}_{\mathrm{D}}$ are the dielectric constant and doping concentration of $\mathrm{SnO}_{2}, \mathrm{~N}_{\mathrm{s}}$ is the surface state density and $\Phi_{\mathrm{bi}}$ is the built-in potential.

If both, the voltage drop in the direct biased junction $\mathrm{V}_{\mathrm{d}}$ and the voltage drop in the nanowire $\mathrm{V}_{\mathrm{NW}}$ are supposed to be small compared with the voltage drop in the reverse biased junction $\mathrm{V}_{\mathrm{i}}$; then, it can be considered, as first approximation, that all the applied voltage falls in this latter junction,

$$
V_{d}+V_{N W} \leq V_{i} \Rightarrow V \approx V_{i}
$$

According to this assumptions, $\ln (\mathrm{I}) \mathrm{vs} \mathrm{V}^{1 / 4}$ should be linear at all temperatures for which the model is valid. Rectifying I-V curve values confirm that the back-to-back Schottky-like assumption can be very useful to describe the electrical response of these $\mathrm{SnO}_{2}$-NWs at room temperature, figure 8 .

The contact resistance presents an activation energy, ie $\mathrm{E}_{\mathrm{a}}=0.27$ $\mathrm{eV}$ in the example shown in the figure 9, which is found depending of the nanowire, but over $200-250^{\circ} \mathrm{C}$ its value becomes, generally, already non predominant. It is important if we keep in mind the gas sensor working temperatures. This behaviour can be explained assuming that some FIB-induced disorder is produced

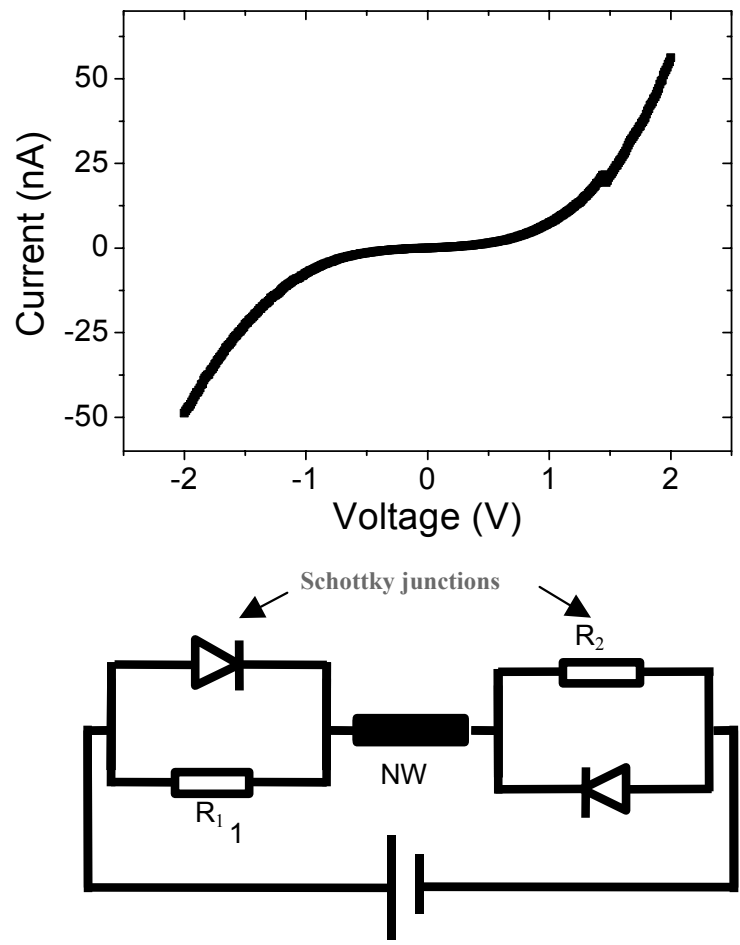

Fig. 7. Non-lineal symmetric $\mathrm{I}(\mathrm{V})$ characteristics in a FIB Pt nanocontact performed on a single nanowires measured using two probes

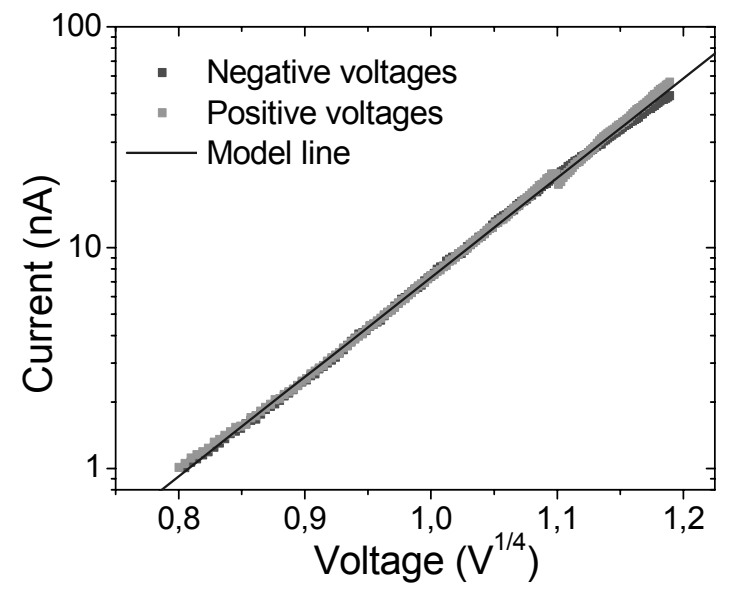

Fig. 8. Logarithm of the current against the $1 / 4$ power of the applied bias according to the model by equations (3-6) 

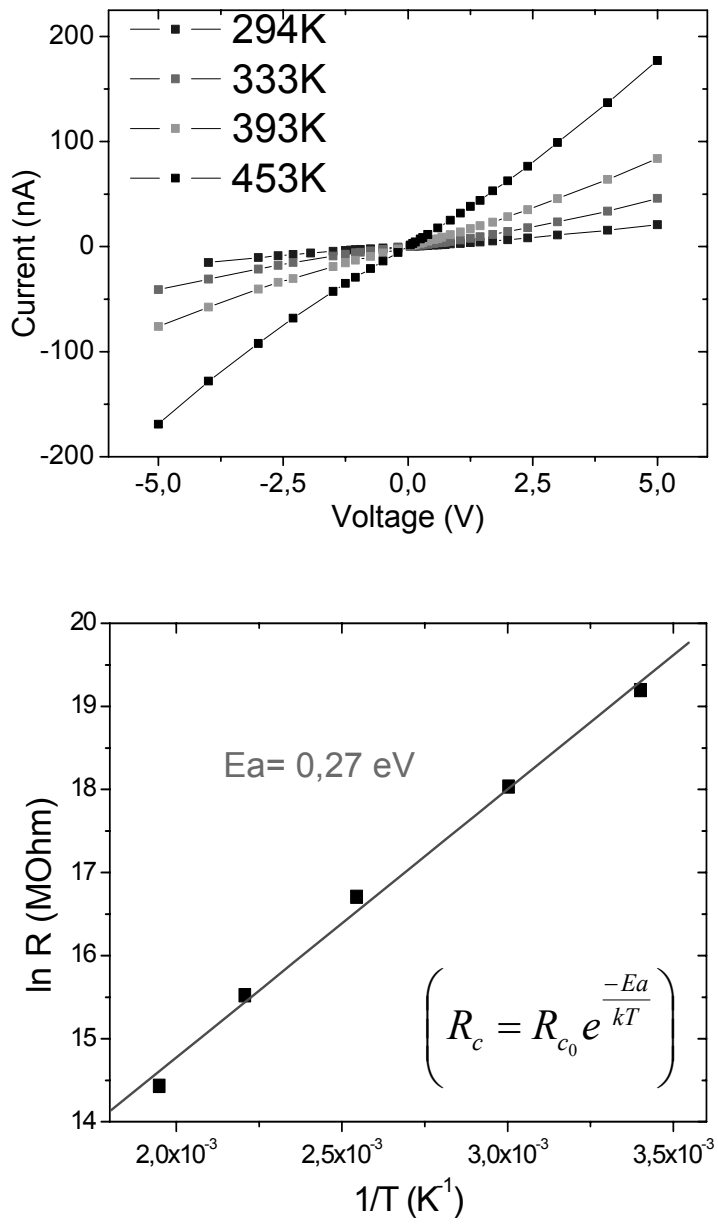

Fig. 9. Dependence of the contact resistance on the temperature

Table 1. Fitted values of equivalent circuit of a single $\mathrm{SnO}_{2}-\mathrm{NW}$ AC Impedance Spectroscopy experiment

\begin{tabular}{|c|c|c|}
\hline $\mathrm{R}(\mathrm{M} \Omega)$ & $\mathrm{Q}(\mathrm{pF})$ & $\mathrm{n}$ \\
\hline $4.315 \pm 0.006$ & $97 \pm 2$ & 0.97 \\
\hline
\end{tabular}

underneath the Pt contacts during the contact fabrication process, creating surface states used by electrons to overcome the barrier, even at low bias, considering multiphonon assisted tunnel mechanisms. Similar sample modifications have already been reported in the past ${ }^{(33)}$ and the increases of the charge barrier transmission capability diminish the non-linearity characteristics up to achieve ohmic performances. Other of the consequences of this contact resistance, which must be taken into account as relevant in many experimental cases, is the heat dissipation.

So, to avoid also the DC thermal effects due to the power dissipation, as well as to avoid any problem with the species diffusion through the structures, we have used AC measurements. AC Impedance Spectroscopy is a well-known and widely used technique in material science studies that can help us to overcome these limitations. Moreover, AC Impedance Spectroscopy is known to be useful to characterise Schottky barriers originated in $\mathrm{Pt}-\mathrm{SnO}_{2}$ junctions.
For the AC measurements we have used an Impedance Analyzer Solartron SI-1260 with a maximum working frequency of $f=5$ $\mathrm{MHz}$ and an Impedance Analyzer Gamry EIS300 with a maximum working frequency of $\mathrm{f}=300 \mathrm{kHz}$. All measurements have been performed inside of a self-made Faraday cage. As far as we know, these are the first attempts of performing four-probe electrical measurement in nanowires contacted with this FIB nanolithography method and using AC impedance spectroscopy to characterise a device based on a single nanowire.

A typical result of the AC Impedance Spectroscopy measurements is shown in figure 10, corresponding to $\mathrm{SnO}_{2} \mathrm{NW}$ of length $\mathrm{L}=7.7 \mu \mathrm{m}$ and diameter $\mathrm{D}=35 \mathrm{~nm}$. The simplest equivalent circuit which fits these experimental data consists of a parallel $\mathrm{RQ}$, where $\mathrm{R}$ is a pure resistance and $\mathrm{Q}$ a CPE (constant phase element). The fitted values (Table 1 ) suggest that $\mathrm{Q}$ is close to an ideal capacitor $(\mathrm{n} \approx 1)$, where $\mathrm{n}$ is the coefficient used to describe the CPE behaviour. The characteristic time $\tau$ of the here-observed charge transport mechanism is $\tau=\mathrm{RC}=0.42 \mathrm{~ms}$.

Similar AC Impedance curves have been observed with other $\mathrm{SnO}_{2}$ NWs. Taking into account that contact resistance at room temperature is much larger than the NW resistance itself in all these samples, we believe that the experimental data are mainly related to the $\mathrm{Pt}-\mathrm{SnO}_{2}$ junction at this temperature rather than the NWs, equation (8a). To have more significant contribution of the nanowire impedance is needed, according to the above discussion, to increase the measurement temperature at least above $200^{\circ} \mathrm{C}$, equation (8b). So this equivalent parallel $R_{T} C_{p}$ circuit is just a simplification of the general circuit shown in the insert of figure 7 where $\mathrm{R}_{\mathrm{T}}$ is dominated by $\mathrm{R}_{\mathrm{C}}$ at room temperature and by $\mathrm{R}_{\mathrm{NW}}$ at enough high temperature. Unfortunately, the capacitance is dominated by the parasitic value introduced by the used silicon wafer substrate which is independent of the applied bias and the measurement temperature. So, any effect of the capacitance contribution of the Schottky barriers has been detected on the total capacitance. A constant value has been measured for bias range from 0 to 8 volts and temperatures from room ambient to $600^{\circ} \mathrm{K}$ at different atmospheres that is attributed to a parasitic component. Describing Schottky barriers of $\mathrm{Pt}-\mathrm{SnO}_{2}$ junctions as a parallel $\mathrm{R}_{\mathrm{C}} \mathrm{C}_{\mathrm{C}}$ circuit has been reported by other groups in the past and, in the present case, it is well-known that both $R_{C}$ and $C_{C}$ values fulfil that:

Low T

$$
R_{N W}<<R_{C} \propto \exp \left(\frac{\phi_{B E}}{k_{B} T}\right) \cong R_{T}
$$

High T

$$
\begin{aligned}
& R_{C}<R_{N W} \cong R_{T} \cdots \cdots . . . \\
& C_{C} \propto\left(\phi_{B E}\right)^{-\frac{1}{2}}<<C_{P} .
\end{aligned}
$$

Where $\Phi_{\mathrm{BE}}$ is the barrier height.

In general, it is quite simple and straightforward to work at the sensing temperature, over $200^{\circ} \mathrm{C}$, applying $\mathrm{AC}$ measurement. Capacitance component becomes related to the parasitic capacitance value and it does not change with the temperature and neither with the applied bias. Usually we are working at 0 volts of DC bias. 


\subsection{Single Nanostructure Based Bottom-up Gas Sensor} Devices With this system we have performed measurements of the nanowire resistance of the bottom-up gas sensor devices, usually, at $265^{\circ} \mathrm{C}$ and at different atmosphere, figure 11.

One of the more interesting features is the analysis against the synthetic air and nitrogen atmospheres. It reveals a clear
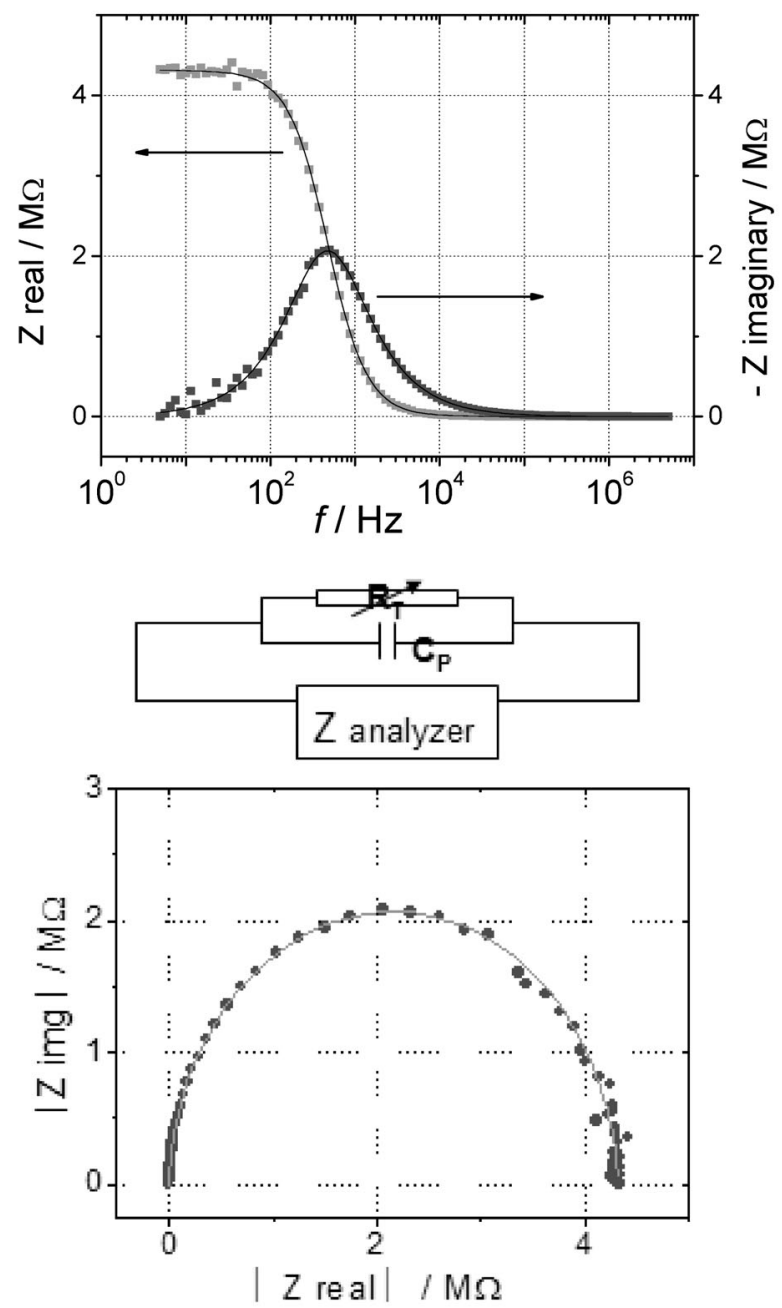

Fig. 10. $\mathrm{AC}$ measurements on a single $\mathrm{SnO}_{2}$ nanowire contacted using focused ion beam

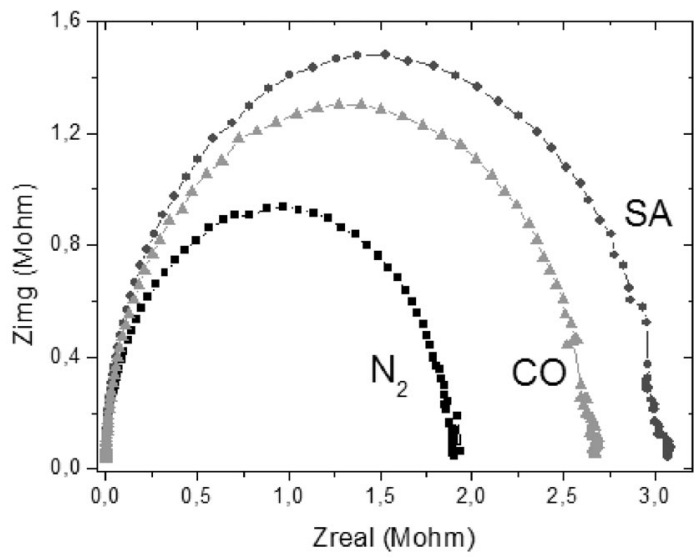

Fig. 11. AC measurement behaviour of a single $\mathrm{SnO}_{2}$ nanowire at different gas atmospheres dependence on the diameter size according to (1/D) dependence, figure 12 and table 2 . It corroborates the existence of a space charge zone created by the adsorbed oxygen which is limiting the central area of the nanowire for electrical transportation.

In order to validate the capability of a single nanowire as individual gas nanosensor, we have performed the measurements of $\mathrm{CO}$ gases, figure 13. In spite of that in the literature there is many papers indicating that has been possible to detect several hundred of $\mathrm{CO}$ ppm or even more, we have been able to detect a concentration as low as $5 \mathrm{ppm}$ of $\mathrm{CO}$ or even less, figure 14. So far we know, it is the lower CO concentration level never detected using a single nanowire or in other words using an individual

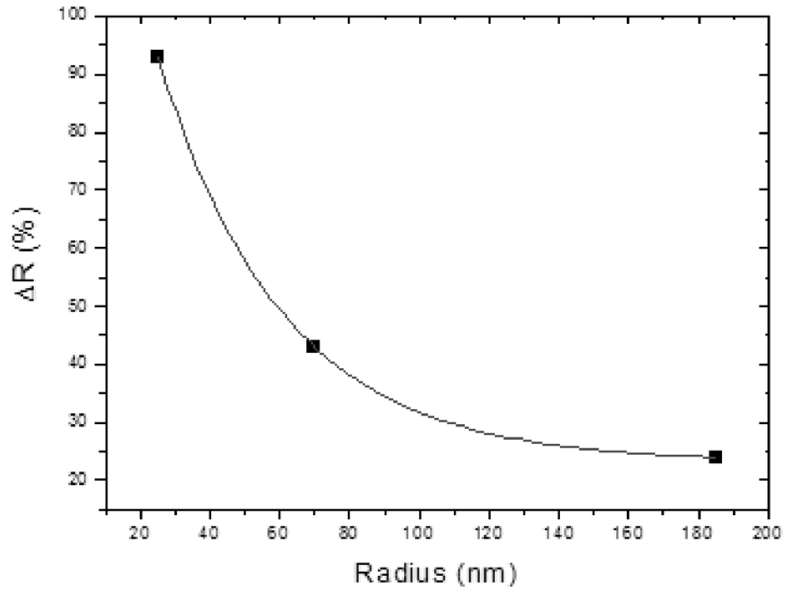

Fig. 12. Dependence on the nanowire radius of the nanowire resistance variation against the nitrogen/synthetic air ambient composition

Table 2. Real impedance component of single nanowires with different radius against nitrogen or synthetic air atmospheres

\begin{tabular}{|c|c|c|}
\hline $\begin{array}{c}\text { Radius } \\
(\mathrm{nm})\end{array}$ & $\begin{array}{c}\text { Real impedande } \\
\text { component under } \\
\text { Nitrogen atmosphere } \\
(\mathrm{M} \Omega)\end{array}$ & $\begin{array}{c}\text { Real impedande } \\
\text { component under } \\
\text { synthetic air } \\
\text { atmosphere }(\mathrm{M} \Omega)\end{array}$ \\
\hline 25 & 1.6 & 3.1 \\
\hline 70 & 1.05 & 1.5 \\
\hline 185 & 0.83 & 1.03 \\
\hline
\end{tabular}

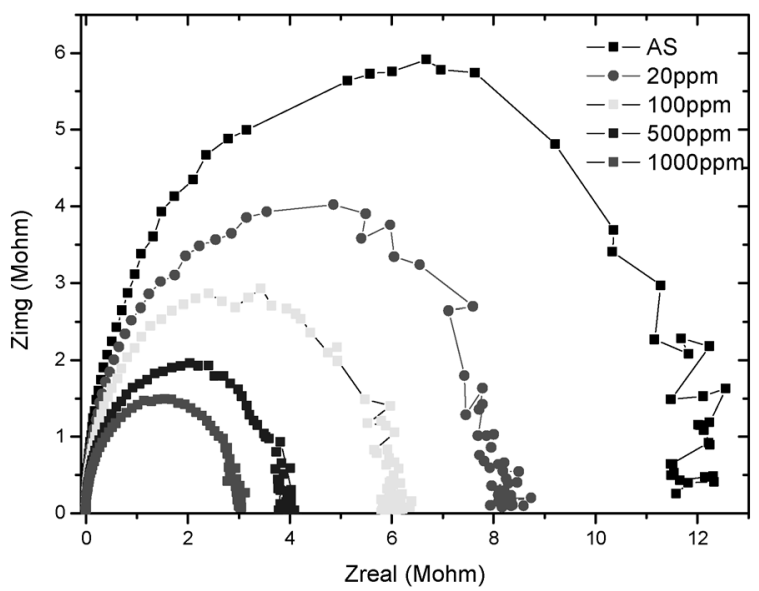

Fig. 13. Impedance measurements of an individual $\mathrm{SnO}_{2}$ nanowire for different $\mathrm{CO}$ concentrations in synthetic air (AS) at $245^{\circ} \mathrm{C}$ 


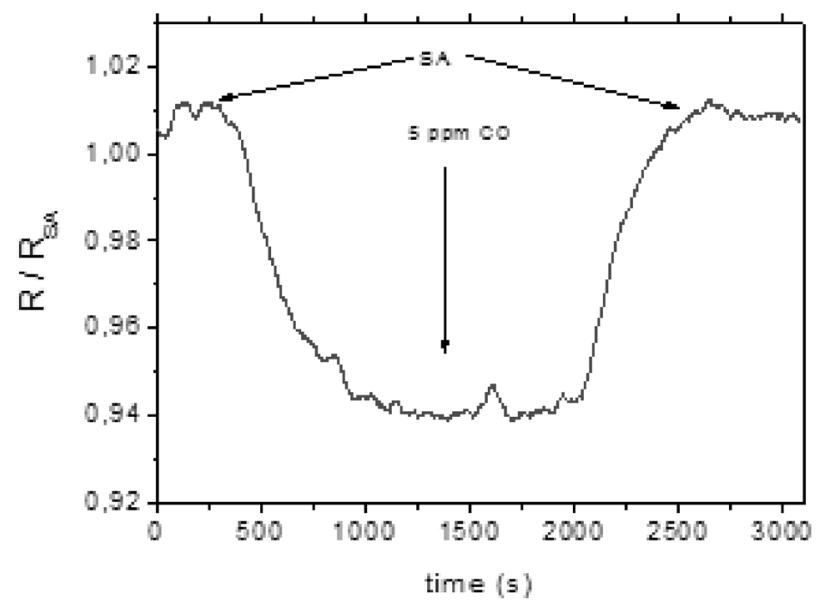

Fig. 14. Single nanowire gas sensor response to $5 \mathrm{ppm}$ of $\mathrm{CO}$ in synthetic air at $295^{\circ} \mathrm{C}$

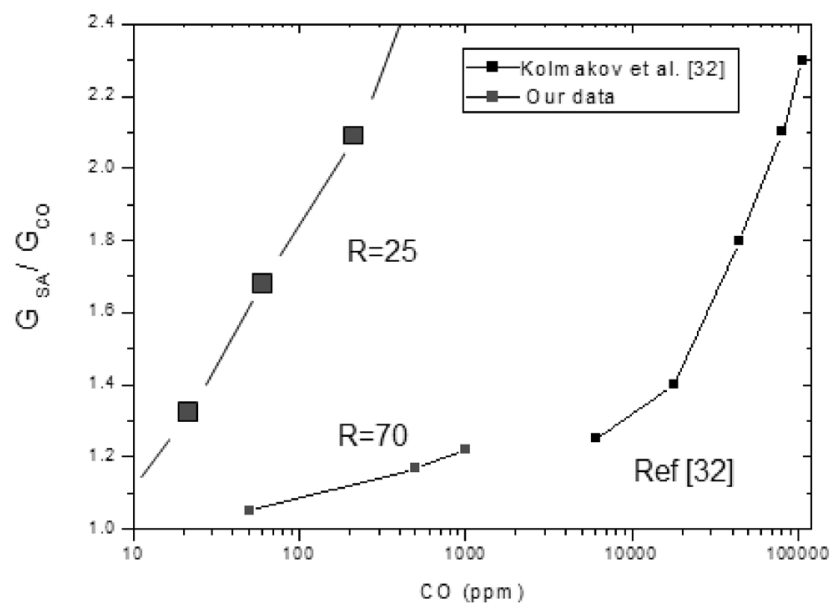

Fig. 15. Sensor response of single $\mathrm{SnO}_{2}$ nanowires with different radius against the $\mathrm{CO}$ concentration

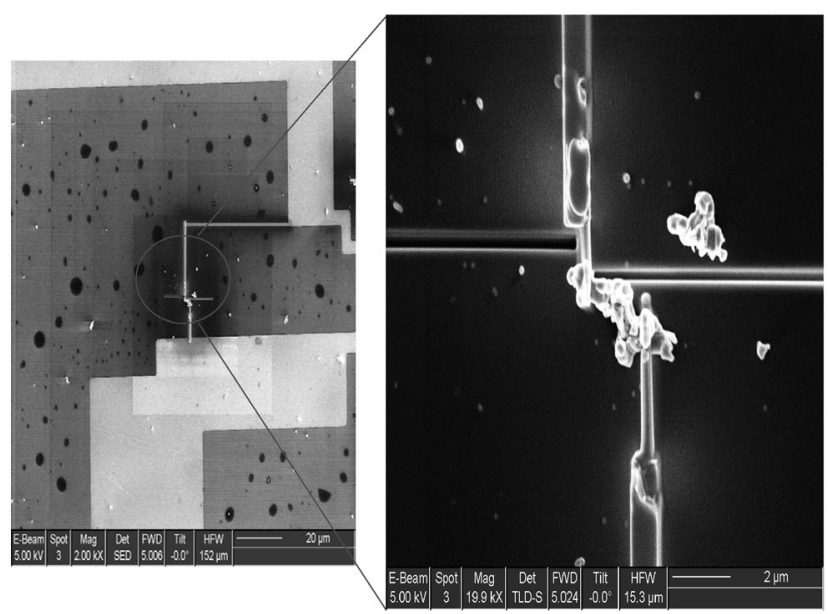

Fig. 16. (Left image) Contacting pattern prepared with both platinum electron- and ion-beam induced deposition between 2 microelectrodes. (Right image) Detailed image of the contacted $3 \mathrm{D} \mathrm{WO}_{3}$ nanocrystals network

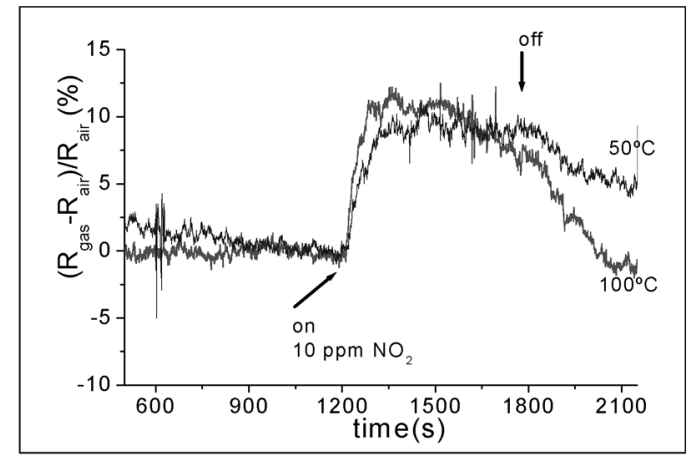

Fig. 17. Electrical response of a $\mathrm{WO}_{3} \mathrm{SBA}$ negative replica in presence of $10 \mathrm{ppm}$ of $\mathrm{NO}_{2}$ at $50^{\circ} \mathrm{C}$ and $100^{\circ} \mathrm{C}$. A similar relative change in resistance for both temperatures is observed $(\Delta \mathrm{R} \approx 10-15 \%)$. Nevertheless, recovery time of initial resistance value is higher at $100^{\circ} \mathrm{C}$ than at $50^{\circ} \mathrm{C}$

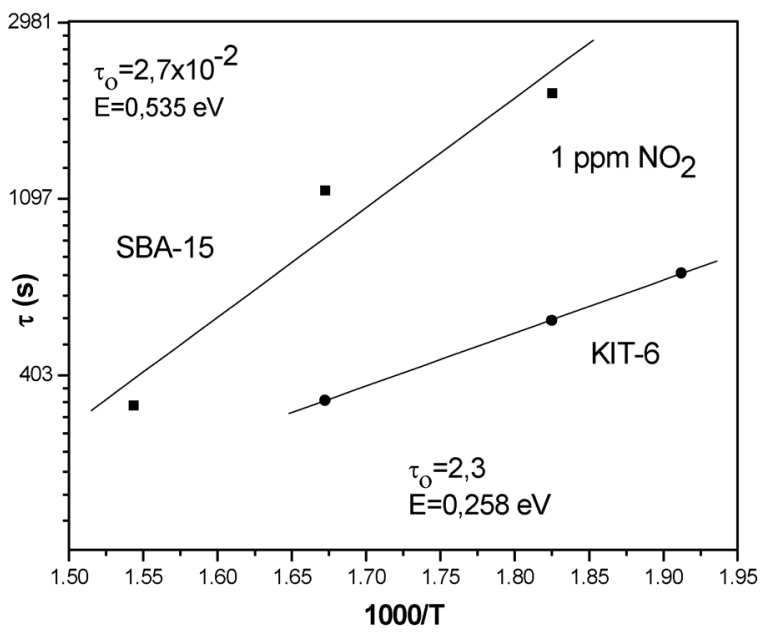

Fig. 18. Thermal dependence of the response time for the $\mathrm{WO}_{3}$ KIT6 and SBA15 negative replica showing different activation energy

nanosensor built bottom up using a single nanowire.

Again, these results are showing the viability of the proposed method and show also the importance of the geometrical parameters of the nanowires. In the figure 15 we show the dependence of the sensor response of individual nanowire on its radius. It points out the importance to use nanowire with diameter smaller than $25 \mathrm{~nm}$ of radius to get outstanding properties as sensors even considering that the single nanowire is presenting the characteristic like a monocrystal. It fits much better with the stability requirements and long term wear-out claims.

This procedure can also be applied to other nanostructures than nanowires. Figure 16 shows the application to $\mathrm{WO}_{3} 3 \mathrm{D}$ nanocrystal networks in order to fabricate gas sensors. This type of sensors suffers relative important changes in the resistance value due to the interaction with $\mathrm{NO}_{2}$ molecules even around room temperature, figure 17. Therefore, in spite of the high contribution of the contact resistance at so low temperatures, it is possible to detect directly the presence of $\mathrm{NO}_{2}$ gas, although it is partially hidden by the contact resistance, $\mathrm{R}_{\mathrm{T}} \sim \mathrm{R}_{3 \mathrm{D}}+\mathrm{R}_{\mathrm{C}}$. 


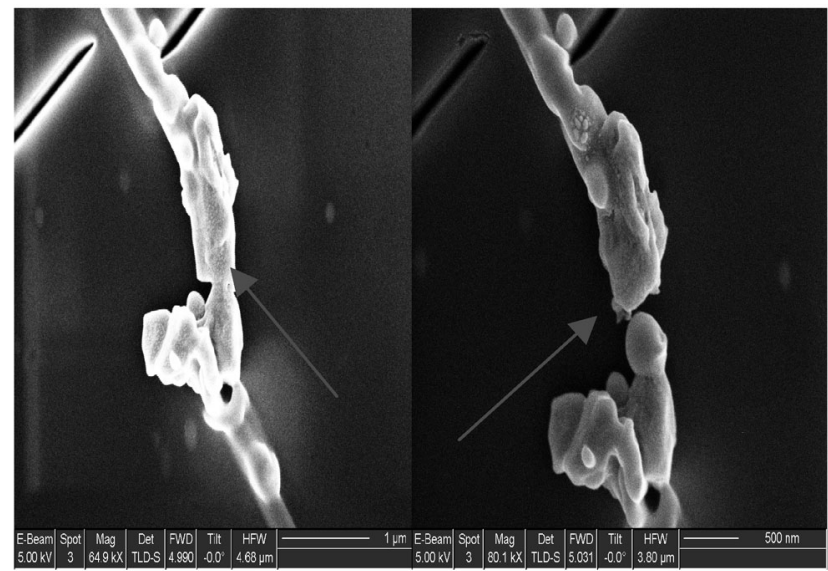

Fig. 19. $\mathrm{WO}_{3}$ SBA negative replica agglomerate before (left) and after (right) applying a current higher than $1 \mu \mathrm{A}$. The agglomerate is destroyed in the junction of 2 groups of nanocrystals (arrows)

Again higher temperatures eliminate the influence of the contact resistance and then experiments under different gas environments can also be used in the study of physical and structural properties of the nanomaterials, specially selecting individual nanowires or simple 3D structrures that can facilitate the mechanisms analysis taking place at the nanocrystal scale. For example, the prepared $\mathrm{WO}_{3}$ KIT- 6 and SBA15 negative replica have different response time with different activation energy such as it is shown in figure 18 that can be studied using the presented procedures and methodology. Nevertheless, it is needed again to underline that the stability and response of these structured can have some problems. Some of them even can be destroyed when high current density flow through them, figure 19, or after long working periods that allow to accumulate degradation for wear-out. For these reasons, further research must be done in order to improve them.

\section{Conclusions}

A procedure for fabricating electrical contacts using a Dual Beam FIB machine between nanometer-sized materials and microelectrodes has been presented. On top and in the proximity of the contacted nanomaterial, the electron beam has been used to perform the deposition process, finishing the remaining part of the contact with the help of ions. In this way, damage introduced by ion bombardment is reduced. Characterization of both ion- and electron-assisted deposited platinum has revealed a high carbon contamination introduced in the fabrication process. These electrical contacts present no degradation after long operation time.

The proposed procedure has been applied to perform electrical contact on single metal oxide nanowires and 3D nanorystal networks. In both cases, the nanostructures are destroyed at current densities lower than the breakdown densities of the deposited platinum. The electrical parameters of the contacted materials are better extracted performing 4-probe electrical measurements in order to avoid contact resistance contribution. Also, low bias is used to eliminate possible heating effect on the structure. Contact resistances have activation energy and, usually, at the gas sensor working temperature range, the resistance becomes less significant than the nanostructure resistance.

Under these conditions, we propose as an outstanding alternative to use AC measurements instead DC one. Furthermore, it demonstrates the viability of fabricating bottom up nanodevices as gas sensors or elements for basic studies based on single nanostructures. Some examples as gas sensors have also been reported and the nanowire size influence determined. Likewise, in spite of that in the literature there is many papers indicating that has been possible to detect several hundred of $\mathrm{CO}$ ppm or even more, we have been able to detect as low as $5 \mathrm{ppm}$ of $\mathrm{CO}$ or even less. So far we know, it is the lower CO concentration level never detected using a single nanowire or, in other words, using an individual bottom up nanosensor based on a single nanowire.

As summary, the developed methodology for building bottom up nanosensor devices from a single nanowires using adequate hotplate or other substrate has allowed us to obtain and characterize very reliable gas nanosensors with excellent and outstanding performances. These characteristics open a lot of activities in the analysis of sensing mechanisms in single nanowires for different atmospheres such as nitrogen, synthetic air, carbon monoxide, humidity, $\mathrm{NO}_{2}$, etc ${ }^{(34) \text {. }}$

\section{Acknowledgment}

These activities have partially been supported by the Spanish project MAGASENS and the EU projects NANOS4 and GOOD FOOD.

(Manuscript received Aug. 30, 2006)

\section{References}

(1) J. Jortner and C. N. R. Rao : Pure Appl. Chem. Vol.74, pp.1489-1783 (2002)

(2) M. Law, J. Goldberger, and P. Yang : Annu. Rev. Mater. Res. Vol.34, No.83, p.122 (2004)

(3) G. J Li and S. Kawi : Matter. Lett. Vol.34, pp.99-102 (1998)

(4) P. A. Smith, C. D. Nordquist, T. N. Jackson, T. S. Mayer, B. R. Martin, J. Mbindnyo, and T. E. Mallouk : Chem.-Eur. J. Vol.8, No.4355 (2002)

(5) M. E. Toimil-Morales, E. M. Höhberger, Ch. Schaeflein, R. H. Blick, R. Neumann, and C. Trautmann : Appl. Phys. Lett. Vol.82, No.2139 (2003)

(6) K. Gamo : Semicond. Sci. Technol. Vol.8, pp.1118-1123 (1993)

(7) S. Reyntjens, R. Puers. and J. Micromech : Microeng. Vol.11, pp.287-300 (2001)

(8) S. Matsui and Y. Ochiai : Nanotechnology Vol.7, pp.247-258 (1996)

(9) D. C. Shaver and B. W. Ward : J. Vac. Sci. Technol. B Vol.4, No.185 (1986)

(10) K. Nikawa : IEICE Trans Fund. Electr. Vol.E77, p.174 (1994)

(11) L. R. Harriot, A. Wagner, and F. Fritz : J. Vac. Sci. Technol. B Vol.4, No.181 (1986)

(12) "Focused ion beam technology, capabilities and applications". FEI Company (2004). Brochure available at (www.feicompany.com)

(13) T. Tao, J. Ro, and J. Melngailis : J. Vac. Sci. Technol. B Vol.8 p.1826 (1990)

(14) J. Puretz and L. W. Swanson : J. Vac. Sci. Technol. B Vol.10, p.2695 (1992)

(15) T. W. Ebbesen, H. J. Lezec, H. Hiura, J. W. Bennett, H. F. Gaemi, and T. Thio : Nature Vol.382, p.54 (1996)

(16) S. B. Cronin, Y-M. Lin, O. Rabin, M. R. Black, J. Y. Ying, M. S. Dresselhaus, P. L. Gai, J-P. Minet, and J-P.Issi : Nanotechnology Vol.13, p.653 (2002)

(17) R. Vajtai et al : Smart. Mater. Struct. Vol.11, pp.691-698 (2002)

(18) K. Xiao et al : Appl. Phys. Lett. Vol.83, No.23 (2003)

(19) J. Ziroff, G. Agnello, J. Rullan, and K. Dovidenko : Mat. Res. Soc. Symp. Proc. Vol.772, M8.8.1 (2003)

(20) G. de Marzi, D.Iacopino, A.J.Quinn, and G.Redmond : J. Appl. Phys. Vol.96, No.6 (2004)

(21) Strata DB 235. Product data sheet. FEI Company.

(22) L. Rotkina, J-F. Lin, and J. P. Bird. Appl. Phys. Lett., Vol.83, p.4426 (2003)

(23) F. Hernandez-Ramirez, O. Casals, J. Rodríguez, A. Vila, A. RomanoRodriguez, J. R. Morante, M. Abid, and S.Valizadeh : S. Mat. Res. Soc. Symp. Proc., J 5.2, (2005)

(24) V. Gopal, V. R. Radmilovic, C. Daraio, S. Jin, P. Yang, and E. Stach, NanoLetters, Vol.4, p.2059 (2004)

(25) A.Vila, F. Hernandez-Ramirez, J. Rodriguez, O. Casals, A. RomanoRodriguez, J. R. Morante, and M. Abid : Materials Science and Engineering C. Vol.26, pp.1063-1066 (2006)

(26) S. Valizadeh, M. Abid, F. Hernandez-Ramirez, A. Romano-Rodriguez, K. 
Hjort, and J. A Schweitz : Nanotechnology, Vol.17, pp.1134-1139 (2006)

(27) E. Comini : Analytica Chimica Acta Vol.568, pp.28-40 (2005)

(28) B. Tian, X. Liu, L. Solovyov, Z. Liu, H. Yang, Z. Zhang, S. Xie, F. Zhang, B. Tu, C. Yu, O. Terasaki, and D. Zhao : J. Am. Chem. Soc. Vol.126, pp.865-875 (2004)

(29) Software used: SRIM, version 2003.26. (www.srim.org)

(30) Y. Zhang, A. Kolmakov, Y. Lilach, and M. Moskovits : J Phys Chem B Vol.109, pp.1923-1929 (2005)

(31) M. Law, H. Kind, B. Messe, F. Kim, and Y. Peidong : Angew Chem, Vol.114, p.2511 (2002)

(32) A. Kolmakov and M. Moskovits : Annu. Rev. Mater. Res., Vol.34, pp.151-180 (2004)

(33) C. Y. Nam, D. Tham, and J. E. Fischer : NanoLetters, Vol.5, pp.2029-2033 (2005)

(34) J. R. Morante, to be published elsewhere.

Francisco Hernández-Ramírez (Non-member) was born in Barcelona

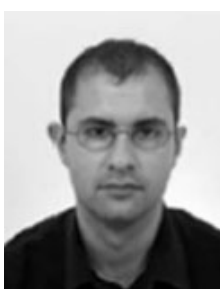
in 1981. He received his degree in physics at the University of Barcelona in 2002. Currently he is student of the doctor's degree program "Electronic Engineering and Technology". His professional experience is related to the use of focused ion beam (FIB) lithography in the fabrication of metal-oxide gas nanosensors.

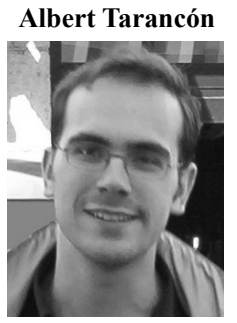

(Non-member) received his Diploma in Physics from the University of Barcelona, Spain, in 2001. From 2002 he is involved in his Ph.D. on the "Strategies for Lowering the Temperature in Solid Oxide Fuel Cells" at the Electronics Department of the University of Barcelona. He is currently an Assistant Professor in the same department. His research interests centre on computer simulations of oxide-ionic conductors, impedance spectroscopy of mixed ionic-electronic conductors and technological aspects of Solid Oxide Fuel Cells.

Albert Romano-Rodríguez (Non-member) obtained his degree (1986)

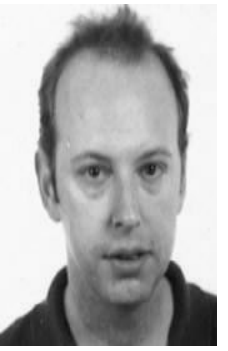
and Ph.D. (1991) in physics from the University of Barcelona. From 1986 to 1988 he worked at the LCMM (pioneer of the Department of Electronics) on electrical, optical and structural characterisation of semiconductor materials and devices. From 1988 to 1991 he was with IMEC (Leuven, Belgium) as a scientific collaborator, where he made all the experimental work of his $\mathrm{PhD}$ dissertation on the characterisation of technological steps for Ultra Large Scale Integration by using Transmission Electron Microscopy and related techniques. Back in Barcelona in 1991, he worked as a researcher at the University of Barcelona and in 1993 he was appointed Professor Titular (lecturer) in electronics in the same university. Since then he has continued his work on the characterisation of semiconducting materials and processes for their fabrication by using different structural, physical and chemical characterisation techniques, moving his interest towards the development of the electronic materials themselves and their application in fields other than microelectronic devices and, more specifically, on solar cells and on different kind of physical and chemical sensors. In this latter field his activity on materials for gas sensors and gas sensing itself are ongoing since 1994. From 2003 he has been strongly involved in the application of Focused Ion Beam techniques in nanotechnology and with strong emphasis in the fabrication of nanocontacts to nanometre-sized materials. He is author or co-author of over 120 scientific and technical papers in these fields and has presented invited talks at different national and international conferences.

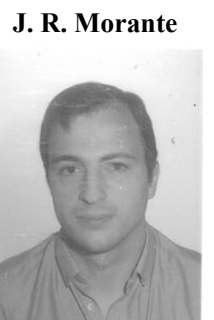

(Non-member) was born in Mataró (Spain). At 1980 he received the $\mathrm{Ph} \mathrm{D}$ degree in Physics from the University of Barcelona. Since 1986 he is full professor of Electronics and director of the Electronic Materials and Engineering group, EME. He has been dean of the Physics Faculty, academic advisor of the Electronic Engineering degree and director of the Electronics Department. Now, he is research head of the EME research group, co-director of the CEMIC (Centre for Engineering of Microsystems) and director of the Centre of Excellence of Advanced materials for Energy and Environment, CeRMAE His activity is devoted to the electronic materials and technology, physics and chemical sensors, actuators, and Microsystems. He has especial interest in nanoscience and nanotechnologies as well as in material for energy and environmental applications.

He has collaborated in international R\&D projects as BRITE, GROWTH (micromechanics, microengineering, gas sensors...), ESPRIT-IST (advanced devices, sensors, actuators, Microsystems, electronic systems,), JOULE (energy related devices and systems), and industrial projects as EUREKA, IBEROEKA and CRAFT

$\mathrm{He}$ is co-author of more than 400 papers in international specialised journal and member of international committees and editorial boards in the field of electronic materials and technology, sensors\&actuators and Microsystems, and material for energy applications. He has distinguished with the research prize Narciso Monturiol from the Generalitat of Catalunya (Spain) and the "Research Distinction "award of Catalonia (Spain). 